http://www.atbp.onaft.edu.ua/

dokladov mezhdunarodnoy konferentsii «Sovremennyie problemyi prikladnoy matematiki i mehaniki: teoriya, eksperiment i praktika», posvyaschennaya 90-letiyu so dnya rozhdeniya akademika N.N. Yanenko. — 2011. — P. 8586

[9] Chalk, C, Borman, D, Sleigh, PA et al. (3 more authors) (Accepted: 2017) A numerical comparison of a frictional and viscoplastic debris flow model. In: to be confirmed. 19th International Conference on Soil Mechanics and Geotechnical Engineering, 17-22 Sep 2017, Seoul, South Korea

[10] Sivetskiy V.I., Sokolskiy O.L., Burla Yu.V. Doslidzhennya protsesu ekstruzIynogo formuvannya polimeriv $z$ urahuvannyam efektu pristInnogo kovzannya. Visnik NTUU "KPI". Himichna Inzheneriya, ekologiya ta resursozberezhennya. 2009. \#1. P. 17-21.

[11] Karvatskiy A. Ya. Reologichni vlastivosti vugletsevih kompozitsIy $v$ diapazoni temperaturi 120-170 ${ }^{\circ} \mathrm{C} / \mathrm{A} . \mathrm{Ya}^{\mathrm{B}}$ Karvatskiy, T. V. Lazarev, D. G. Shvachko, O. S. Tischenko // Visnik NatsIonalnogo tehnIchnogo unIversitetu "HarkIvskiy polItehnIchniy Institut". SerIya : NovI rIshennya v suchasnih tehnologIyah. - 2016. - \# 18. - p. $74-79$.

[12] Lazarev T. V. Matematicheskaia model' protsessa ekstruzii viazko-plastichnoi uglerodnoi massy / T. V. Lazarev, A. Ia. Karvatskii, S. V. Leleka, A. Iu. Pedchenko // Visnik Natsional'nogo tekhnichnogo universitetu "Kharkivs'kii politekhnichnii institut". Seriia : Novi rishennia v suchasnikh tekhnologiiakh. - 2016. - № 12. - P. 31-37.

\title{
МОДЕЛИРОВАНИЕ БАЛАНСИРОВКИ ЭНЕРГОСИСТЕМЫ ПРИ УСЛОВИИ СОДЕРЖАНИЯ В НЕЙ ЗНАЧИТЕЛЬНОЙ ДОЛИ НЕДИСПЕТЧЕРИЗУЕМЫХ ИСТОЧНИКОВ ЭНЕРГИИ
}

\author{
Максимов М. М. ${ }^{1}$, Давидов В. О. ${ }^{2}$ \\ ${ }^{1,2}$ Одесский национальный политехнический университет, Одесса \\ ORCID: ${ }^{1} 0000-0002-5626-5265,{ }^{2} 0000-0003-3099-7596$ \\ Email: ${ }^{1}$ maximov.agro@gmail.com, ${ }^{2}$ davydov@opu.ua
}

Copyright (C) 2018 by author and the journal "Automation of technological and business - processes. This work is licensed under the Creative Commons Attribution International License (CC BY). http://creativecommons.org/licanses/by/4.0

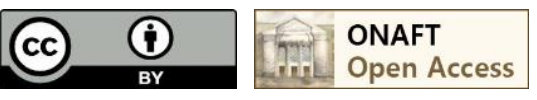

DOI:

\footnotetext{
Аннотация.

Повышение доли недиспетчеризуемых возобновляемых источников энергии в единых энергосистемах влечет за собой проблему балансировки энергосистемы. Как правило, затраты на поддержание баланса между произведенной и потребленной энергией сторонники «зеленой» энергии перекладывают на плечи энергосистемы и не учитывают при подсчете экономического эффекта от возобновляемых источников.

Цель данной работы, провести имитаџионное моделирование процесса балансировки энергосистемь с больщой долей недиспетчеризуемых возобновляемых источников.

В работе проведен анализ пусковых режимов различных типов энергоустановок. Смоделирована энергосистема с различным соотношением диспетчерезуемых энергоустановок. Проведена оценка различных сценариев развития событий при появлении дефицита генерируемой энергии в размере 25\%, 50\% и 75\% от установленной мошности недиспетчерезуемых источников энергии. Показано, что традиционный метод компенсации возмущений за счет вращающчегося резерва при увеличении доли возобновляемых источников не может полностью компенсировать эти возмущения, что на практике приведет к отключению от энергосети части потребителей.

Повышение доли недиспетчеризуемых возобновляемых источников в единой энергетической системе ведет $к$ уменьшению доли обычных энергоустановок, которые потенџиально могут поддерживать баланс.

Нормализовать работу энергосистемы можно путем дублирования мощности недиспетчеризуемых источников эквивалентной мощностью источников с мальм временем пуска, например, дизель-генераторами. Такой подход
} 
http://www.atbp.onaft.edu.ua/

позволяет полностью компенсировать все возмущения в системе, но его стоимость должна учитываться при анализе экономической эффективности возобновляемых источников.

Abstract.

An increase in the share of non-dispensable renewable energy sources in unified energy systems entails the problem of balancing the energy system. As a rule, the supporters of "green" energy shift the costs of maintaining the balance between the energy produced and consumed to the shoulders of the energy system and do not take into account when calculating the economic effect of renewable sources.

The purpose of this work is to conduct a simulation of the process of balancing the power system with a large share of nondispensable renewable sources.

The paper analyzes the starting conditions of various types of power plants. A power system with a different ratio of dispensable power plants is simulated. Various scenarios for the development of events with the occurrence of a deficit of generated energy in the amount of 25\%, 50\% and $75 \%$ of the installed capacity of non-dispensable energy sources were evaluated. It is shown that the traditional method of compensating for disturbances due to a rotating reserve with an increase in the share of renewable sources cannot fully compensate for these disturbances, which in practice will lead to disconnection of some consumers from the power grid.

An increase in the share of non-dispensable renewable sources in a single energy system leads to a decrease in the share of conventional power plants that can potentially maintain balance.

The operation of the power system can be normalized by duplicating the power of non-dispensable sources with the equivalent power of sources with a short start-up time, for example, by diesel generators. This approach allows you to fully compensate for all disturbances in the system, but its cost should be taken into account when analyzing the economic efficiency of renewable sources.

Ключевые слова: недиспетчеризуемые возобновляемые источники энергии, баланс энергосистемы, моделирование.

Keywords: non-dispensable renewable energy sources, energy system balance, modeling.

Введение. Сегодня бытует мнение, что возобновляемые источники энергии (ВИЭ), в частности солнечные и ветровые электростанции, производят дешевую энергию и могут решить энергетические проблемы промышленно развитых стран. В качестве примеров часто приводят Данию в которой доля ВИЭ составляет 35\%. При этом умалчивают, что Дания не имеет развитой промышленности и производит энергии в 3,5 раза меньше Украины [1, 2]. Также удачное географическое расположение Дании позволяет получать высокий КИУМ (42\%) на офшорных ветровых электростанциях [3].

Различные источники часто приводят в качестве примера Германию как европейского лидера по внедрению ВИЭ. При этом умалчивают, что в целом энергетика Германии находится в катастрофическом состоянии [4]. Ежедневно по всей стране происходит около 500 отключений энергии. Экономика несет огромные убытки. Стоимость электроэнергии возросла. Количество выбросов углекислого газа не уменьшилось, а возросло, хотя переход на «зеленую» энергию проходил под лозунгами о борьбе за окружающую среду и снижение вредных выбросов. Оставшиеся от предыдущей энергосистемы генерирующие мощности не справляются с балансировкой пилы нагрузки. В результате ежедневно наблюдаются как кризисы перепроизводства электроэнергии, так и недопроизводства.

Основная причина такого пагубного влияния ВИЭ на энергосистемы заключается в том, что большинство ВИЭ относятся к классу недиспетчеризуемых. Самыми популярными сегодня являются солнечные и ветровые электростанции. Количеством вырабатываемой на таких станциях электроэнергией невозможно управлять. Более того, это количество существенно зависит от случайных факторов, таких как: уровень и характеристики облачности, сила ветра, количество пыли на солнечных панелях.

Анализ последних исследований и публикаций. Создание единых энергосистем (ЕЭС) дает как значительные технические и экономические плюсы, так и определенные минусы. Детально проблемы ЕЭС описаны в работе [5]. Кратко поясним основные моменты.

Один из главных минусов ЕЭС касается аварийных ситуаций, когда толчки нагрузки или токи короткого замыкания в одной точке системы воздействуют на оборудование всей ЕЭС.

Для локализации подобных аварий прибегают к крайней мере - отключению ряда потребителей. Подобную противоаварийную автоматику называют аварийной частотной разгрузкой (АЧР). Она широко используется в энергетических системах и за долгие годы эксплуатации достаточно хорошо отработана.

Причина такого радикального подхода заключается в том, что отключение потребителей можно осуществить практически мгновенно, в то время как включение резервных мощностей требует времени.

Создание ЕЭС привнесло определенную специфику в процесс развития аварии при дефиците генерации. Аварийный дефицит генерации возникает в отдельной энергосистеме, что приводит к резкому изменению обменных мощностей и потере устойчивости параллельной работы. Межсистемные связи отключаются и еще больше нарушают баланс активной мощности в аварийной энергосистеме. 
Основной принцип современной разгрузки - существенное увеличение числа очередей. Чем больше число очередей и, следовательно, чем меньше нагрузка, отключаемая каждой очередью, тем более гибкой становится вся система разгрузки.

«Самонастройка» разгрузки, кроме выполнения ее большим числом очередей, достигается также разбиением всех устройств на несколько категорий:

- АЧР-1 - быстродействующая (до 5 с) разгрузка, имеющая различные уставки по частоте;

- АЧР-2 - медленнодействующая (10 20 c) разгрузка с близкими уставками по частоте и различными уставками по времени;

- дополнительная - действующая при больших дефицитах мощности и предназначенная для ускорения отключения потребителей и увеличения объема отключаемой нагрузки.

Верхние уставки АЧР-1 и АЧР-2 принимаются близкими к 49 - 49,3 Гц, чтобы отключение потребителей происходило после того, как будет реализован вращающийся резерв мощности, и чтобы подъем частоты обеспечил возможность длительной надежной работы турбоагрегатов и электростанций.

В нормальном режиме агрегаты электростанций работают не всегда с полной нагрузкой. В таком случае они способны при необходимости увеличить свою мощность. Это так называемый вращающийся резерв. Величина его зависит от графика нагрузки и времени суток и может составлять $10 \div 15 \%$ и более от номинальной мощности агрегатов [5].

Резервные агрегаты могут находиться в остановленном состоянии. Под таковыми следует понимать те агрегаты, которые сравнительно быстро смогут быть пущены в ход, синхронизированы с системой и принять на себя нагрузку.

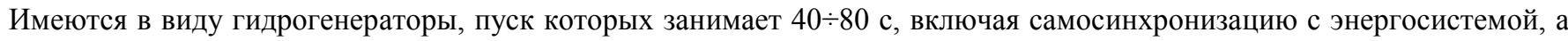
также гидрогенераторы, работающие в режиме синхронного компенсатора. В аварийной ситуации, сопровождающейся дефицитом генерации, эти гидрогенераторы переводятся в режим с выдачей активной мощности.

Очевидно, что чем крупнее ЕЭС, тем больше возможностей по компенсации дефицита энергии при разбалансе системы. Практика показывает, что ЕЭС Украины вполне справляется с колебаниями нагрузки в пределах 5\%. Но что произойдет если доля ВИЭ будет расти? Ведь увеличение доли ВИЭ ведет к уменьшению доли обычных электростанций, главное достоинство которых - это диспетчерезуемость т.е. возможность управлять количеством производимой электроэнергией. Управлять производимой солнечными и ветровыми станциями энергией невозможно. Очевидно, что рост доли ВИЭ потенциально снижает возможности системы поддерживать энергетический баланс.

Сейчас энергопотребление Украины составляет примерно 18 ГВт [6]. Суммарная установленная мощность ВИЭ на октябрь 2019 года составляет 5 ГВт [7]. Реально, на пике, ВИЭ вырабатывают 1,8 ГВт. Что в 3 раза меньше установленной мощности. И это еще одна проблема. Разница в сезонной выработке электроэнергии зимой и летом на СЭС отличается почти в 5 раз. На примере Германии видно, что в солнечные ветреные летние дни, выработка электроэнергии превышает ее потребление и Германия вынуждена платить другим странам, чтобы они утилизировали ее излишки энергии. Получается экономический парадокс. Страна тратит ресурсы на производство «зеленой» энергии, а затем платит другим странам, чтобы у нее эту энергию забрали и все это под лозунгом дешевой «зеленой» энергии.

Целью статьи является разработка и исследование упрощенной модели управления структурой энергетических установок энергосистемы при нанесении возмущения, которое характеризуется случайным отключением недеспечеризируемых установок, при условии содержания в энергосистеме значительной доли ВИЭ.

Исходные данные. Согласно различным прогнозам к 2030 году суммарная мощность ВИЭ в Украине достигнет 12 ГВт.

Рассмотрим гипотетическую энергосистему, состоящую из следующих энергетических установок:

- 10 дизель-генераторных установок (ДГУ) мощностью 5 МВт каждая;

- 5 ГЭС мощностью 800 МВт каждая;

- 6 газотурбинных установок (ГТУ) работающих на газе мощностью 1000 МВт каждая;

- 10 паротурбинных установок (ПТУ) работающих на угле мощностью 2000 МВт каждая;

- 3 АЭС мощностью 1000 МВт каждая;

— ВИЭ суммарной мощностью 9000 МВт.

Для разработки модели поведения ЕЭС были проанализирован ряд положений.

Алгоритм работы АЧР-1 и АЧР-2 позволил определить время срабатывания соответствующих защит. Для АЧР-1 время срабатывания было принято 5 с. Для АЧР-2 - 20 с.

Анализ пусковых режимов различных типов энергоустановок позволил определить среднее время запуска из холодного и горячего состояния и время перехода с одной мощности на другую (табл. 1).

Также был проведен анализ средней стоимости эксплуатации различных типов энергоустановок: ДГУ -

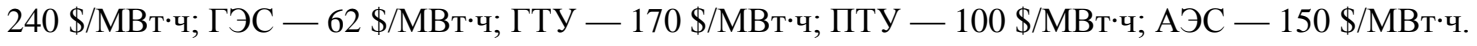


Таблица 1 - Временные характеристики пусковых режимов различных типов енергоустановок

\begin{tabular}{|c|c|c|c|}
\hline Тип энергоустановки & $\begin{array}{c}\text { Время запуска из } \\
\text { холодного состояния, с }\end{array}$ & $\begin{array}{c}\text { Время запуска из } \\
\text { горячего состояния, с }\end{array}$ & $\begin{array}{c}\text { Время перехода на } \\
\text { новую мощность, с }\end{array}$ \\
\hline ДГУ & 1 & 1 & 3 \\
\hline ГЭС & 90 & 420 & 3 \\
\hline ГТУ & 900 & 9000 & 3 \\
\hline
\end{tabular}

\section{Алгоритм моделирования.}

Алгоритм моделирования должен был определить такие величины как:

- суммарное количество недополученной потребителями энергии $E_{l o s t}$, МВт·ч;

— текущие затраты $C(t)$ на функционирование всех энергоустановок, \$;

- текущие удельные затраты $C_{f u n c}(t)$ на функционирование всех энергоустановок, \$/MВт·ч;

- суммарные затраты $C_{f u n c}$ на функционирование всех энергоустановок, \$.

Величина $E_{l o s t}$ определяется как:

$$
E_{l o s t}=\sum_{t=0}^{T} N_{d i s c}(t) / 3.6[\mathrm{MBT} \cdot ч]
$$

где: $N_{d i s c}(t)$ - отключенная в момент времени $t$ мощность потребителей, МВт.

$T$ - интервал моделирования, с.

Величина $C_{f u n c}(t)$ определяется как:

$$
C_{f u n c}(t)=\frac{\sum C_{i}(t)}{\sum N_{i}(t)}
$$

где: $C_{i}(t)$ - текущие затраты на функционирование $i$-й установки, $\$$;

$N_{i}(t)$ - текущая мощность $i$-й установки, МВт;

В ходе моделирования включение и переход с одной мощности на другую для различных энергоустановок должен осуществляться в соответствии с данными табл. 1.

Работа над алгоритмом, позволяющим смоделировать поведение энергосистемы, показала, что при кажущейся свое простоте он представляет сложную многоуровневую систему проверок и условий.

В основе алгоритма лежит бесконечный цикл. Изменение состояний системы рассматривается с шагом в 1 с. В обобщенном виде алгоритм можно представить следующим образом.

Шаг 1. Если обнаружена авария, т.е. производство энергии меньше потребления более чем на $5 \%$, то перейти в состояние аварии и запустить таймеры срабатывания АЧР-1 и АЧР-2.

Шаг 2. Если наступило время срабатывания АЧР-1 отключить часть потребителей. Отключенная мощность принимается равной половине текущего дефицита мощности (с учетом запущенных резервов).

Шаг 3. Если наступило время срабатывания АЧР-2 отключить оставшуюся часть потребителей. Отключенная мощность принимается равной всему текущему дефициту мощности.

Шаг 4. Если система находится в состоянии аварии, то подсчитать суммарную запускаемую мощность установок. Иначе перейти к шагу 15.

Шаг 5. В цикле перебрать все энергетические установки. Если установка имеет вращающийся резерв, то перевести установку в состояние разгона. Увеличить суммарную запускаемую мощность на величину вращающегося резерва текущей установки.

Шаг 6. Если суммарная запускаемая мощность больше дефицита энергии, либо достигнут конец цикла, то перейти шагу 7. Иначе перейти к шагу 5.

Шаг 7. В цикле перебрать все энергетические установки. Если время запуска установки меньше планируемого времени аварии и если установка отключена либо находится в состоянии горячего резерва, то перевести установку в состояние включения. Увеличить суммарную запускаемую мощность на величину потенциальной мощности текущей установки. 
http://www.atbp.onaft.edu.ua/

Шаг 8. Если суммарная запускаемая мощность больше дефицита энергии, либо достигнут конец цикла, то перейти шагу 9. Иначе перейти к шагу 7.

Шаг 9. В цикле перебрать все энергетические установки. Если установка в состоянии разгона перейти к шагу 10. Если установка в состоянии включения перейти к шагу 13.

Шаг 10. Если завершилось время ожидания разгона установки, рассчитать величину $\Delta N$ равную разности между номинальной и текущей мощностью установки.

Шаг 11. Если текущий дефицит энергии больше либо равен величине $\Delta N$, то уменьшить суммарную запускаемую мощность на величину $\Delta N$. Увеличить текущую генерируемую мощность на величину $\Delta N$. Уменьшить величину отключенной мощности потребителей на величину $\Delta N$. Установить текущую мощность установки равной номинальной.

Шаг 12. Если текущий дефицит энергии меньше величины $\Delta N$, то уменьшить суммарную запускаемую мощность на величину текущего дефицита энергии. Увеличить текущую генерируемую мощность на величину текущего дефицита энергии. Уменьшить величину отключенной мощности потребителей на величину текущего дефицита энергии. Увеличить текущую мощность установки на величину текущего дефицита энергии.

Шаг 13. Если завершилось время ожидания пуска установки повторить шаги 9-12 для запускаемых установок.

Шаг 14. Если дефицит энергии компенсирован, то перейти в состояние замещения источников. Иначе перейти к шагу 2.

Шаг 15. Если система находится в состоянии замещения, то перейти к шагу 16. Иначе перейти к шагу 2.

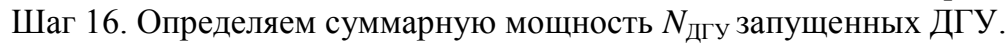

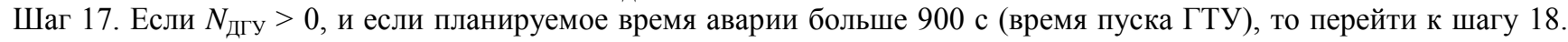
Иначе перейти к шагу 23.

Шаг 18. В цикле перебрать все энергетические установки. Если установка является ГТУ и если установка отключена либо находится в состоянии горячего резерва, то перевести установку в состояние включения. Увеличить суммарную запускаемую мощность на величину потенциальной мощности текущей установки.

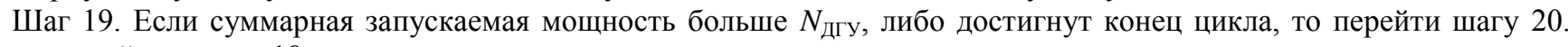
Иначе перейти к шагу 18.

Шаг 20. Если завершилось время ожидания пуска установки, рассчитать величину $\Delta N$ равную разности между номинальной и текущей мощностью установки.

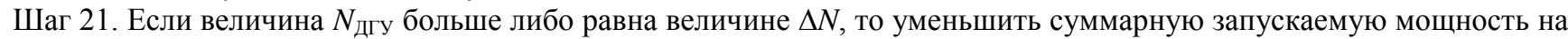
величину $\Delta N$. Установить текущую мощность установки равной номинальной. Отключить ДГУ и при необходимости уменьшить текущую мощность ДГУ так чтобы уменьшаемая мощность ДГУ была равна $\Delta N$.

Шаг 22. Если величина $N_{\text {дгу меньше величины }} \Delta N$, то уменьшить мощность текущей установки на величину $N_{\text {дгу. }}$ Отключить все ДГУ.

Шаг 23. Если планируемое время аварии больше 14000 с (время пуска ПТУ), то выполнить шаги 18-22 применительно к ГЭС замещаемых ПТУ.

Шаг 24. В цикле перебрать все энергетические установки. Определить текущую суммарную стоимость работы системы, суммарную генерируемою энергию и удельную стоимость эксплуатации системы.

Шаг 25. Вывести данные в протокол и перейти к шагу 2.

Чтобы исключить самопроизвольный переход на самый дешевый источник энергии (ПТУ - 100\$/ МВт·ч) некоторые установки помечаются определенным признаком, что позволяет исключить их из очереди на замещение.

Результаты моделирования. Определим исходное состояние системы. Все ДГУ отключены. Все ГЭС находятся в состоянии горячего резерва. АЭС создают базовую нагрузку 3 ГВт. 2 ГТУ и одна ПТУ работают на 90\% мощности (10\% вращающийся резерв). Одна ПТУ работает на мощности 400 МВт. Суммарная мощность ВИЭ составляет 9 ГВт. Суммарная генерируемая мощность ЕЭС составляет 16 ГВт.

Будем считать, что изначально продолжительность возмущения определена как 120 с. На 100 секунде оператор получает информацию, что длительность аварии будет 1200 с, а на 1100 секунде оператор получает дополнительную информацию об увеличении длительности возмущения до 16000 с. Таким образом, общий временной интервал моделирования $T$ составлял 16000 с.

Для начала была оценена работа системы в безаварийном режиме. Общие затраты на функционирование системы за время $T$ составили 4337510 \$. Удельная стоимость составила 141 \$ МВт·ч.

Далее был рассмотрен ряд сценариев, при которых наносится возмущение, и доля генерируемой ВИЭ энергии уменьшается на 25\%, 50\% и 75\%, а баланс в системе поддерживался только за счет отключения потребителей. Очевидно, что, так как, новые установки не запускаются, стоимость эксплуатации в этих случая составляет те же 4337510 \$ но количество энергии, которую недополучил конечный потребитель соответственно равно 10,20 и 30 ГВт•ч. Особого интереса эти сценарии не представляют и были проанализированы только для полноты картины.

Далее был рассмотрен ряд сценариев, при которых генерируемая ВИЭ энергия уменьшается на 25\%, 50\% и 75\%, а энергосистема пытается компенсировать дефицит энергии всеми возможными способами.

В первом сценарии дефицит энергии на момент аварии составил 2250 МВт.

Ниже приведены выдержки из протокола работы системы.

1 секунда. Активированы АЧР-1 и АЧР2. Подготовка к переводу блоков ГТУ1, ГТУ2, ПТУ1, ПТУ2 на новую мощность. Подготовка к запуску блоков ДГУ1 - ДГУ 10 и ГЭС1. Суммарная запускаемая мощность 2850 МВт. 
3 секунда. Запущены из холодного состояния блоки ДГУ1 - ДГУ10. Дефицит энергии 2200 МВт.

5 секунда. Блоки ГТУ1, ГТУ2, ПТУ1 и ПТУ2 переведены на максимальную мощность. Дефицит энергии: 200 МВт.

7 секунда. Срабатывание АЧР-1. Отключено 100 МВт.

17 секунда. Запущен из горячего резерва блок ГЭС1. Мощность (макс/тек) 800/200. Дефицит энергии 0. Подключены все отключенные ранее потребители.

Подготовка к переводу блока ГЭС1 на новую мощность для замещения.

21 секунда. Блок ГЭС1 переведен на мощность 250 МВт. Отключены блоки ДГУ1 - ДГУ 10.

22 секунда. Срабатывание АЧР-2. Отключено 0 МВт.

100 секунда. (Ожидаемое время аварии увеличилось до 1200 c). замещения.

1001 секунда. Запущен блок ГТУ3 на мощность 250 МВт. Отключен блок ГЭС1.

1100 секунда. (Ожидаемое время аварии увеличилось до 16000 с). $\quad$ Подготовка к пуску блока ПТУ3 для замещения.

13701 секунда. Запущен блок ПТУ3 на мощность 250 МВт. Отключен блок ГТУ3.

Потребитель недополучил: 277.778 кВт·ч.

Затрачено: 5463940 \$.

Аналогичным образом были проанализированы сценарии для 50\% и 75\% уменьшения выработки энергии ВИЭ. Дополнительно были смоделированы сценарии, при которых в системе отсутствуют ДГУ и ГЭС, а также отсутствует вращающийся резерв. Отдельно был смоделирован сценарий, при котором установочная мощность ВИЭ дублируется эквивалентной мощностью дизель-генераторных установок. Результаты сведены в табл. 2.

На основании результатов моделирования была исследована динамика изменения текущей и удельной стоимости функционирования системы (рис. 1,2 ).

Таблица 2 - Сводные результаты моделирования различных сценариев

\begin{tabular}{|c|c|c|}
\hline Сценарий & $\begin{array}{c}\text { Количество } \\
\text { недополученной } \\
\text { потребителем } \\
\text { энергии, кВт·ч }\end{array}$ & $\begin{array}{c}\text { Стоимость } \\
\text { эксплуатации } \\
\text { системы, \$ }\end{array}$ \\
\hline Нормальный режим работы & 0 & 4337510 \\
\hline $\begin{array}{c}\text { Выработка ВИЭ уменьшается на 25\%, компенсация за счет отключения } \\
\text { потребителей }\end{array}$ & 10000000 & 4337510 \\
\hline $\begin{array}{c}\text { Выработка ВИЭ уменьшается на 50\%, } \\
\text { компенсация за счет отключения потребителей }\end{array}$ & 20000000 & 4337510 \\
\hline $\begin{array}{c}\text { Выработка ВИЭ уменьшается на } 75 \%, \\
\text { компенсация за счет отключения потребителей }\end{array}$ & 30000000 & 4337510 \\
\hline Выработка ВИЭ уменьшается на 25\%, компенсация за счет резервов & 278 & 5463940 \\
\hline Выработка ВИЭ уменьшается на 50\%, компенсация за счет резервов & 3402 & 6995180 \\
\hline Выработка ВИЭ уменьшается на 75\%, компенсация за счет резервов & 414445 & 8558110 \\
\hline $\begin{array}{c}\text { Выработка ВИЭ уменьшается на 25\%, компенсация за счет резервов. } \\
\text { Отсутствуют ДГУ }\end{array}$ & 347 & 5463840 \\
\hline $\begin{array}{c}\text { Выработка ВИЭ уменьшается на 75\%, компенсация за счет резервов. } \\
\text { Отсутствуют ДГУ }\end{array}$ & 428111 & 8521680 \\
\hline $\begin{array}{c}\text { Выработка ВИЭ уменьшается на 25\%, компенсация за счет резервов. } \\
\text { Отсутствуют ГЭС }\end{array}$ & 671368 & 7351070 \\
\hline $\begin{array}{c}\text { Выработка ВИЭ уменьшается на 75\%, компенсация за счет резервов. } \\
\text { Отсутствуют ГЭС }\end{array}$ & 1037930 & 8548790 \\
\hline $\begin{array}{c}\text { Выработка ВИЭ уменьшается на 25\%, компенсация за счет резервов. } \\
\text { Отсутствует вращающийся резерв }\end{array}$ & 3055 & 5935740 \\
\hline $\begin{array}{c}\text { Выработка ВИЭ уменьшается на 75\%, компенсация за счет резервов. } \\
\text { Отсутствует вращающийся резерв }\end{array}$ & 961320 & 8255290 \\
\hline $\begin{array}{c}\text { Выработка ВИЭ уменьшается на 25\%. 100\% первоначального дефицита } \\
\text { компенсируется ДГУ }\end{array}$ & 0 & 5942560 \\
\hline $\begin{array}{c}\text { Выработка ВИЭ уменьшается на 50\%. 100\% первоначального дефицита } \\
\text { компенсируется ДГУ }\end{array}$ & 0 & 7352280 \\
\hline $\begin{array}{c}\text { Выработка ВИЭ уменьшается на 75\%. 100\% первоначального дефицита } \\
\text { компенсируется ДГУ }\end{array}$ & 0 & 8171600 \\
\hline
\end{tabular}




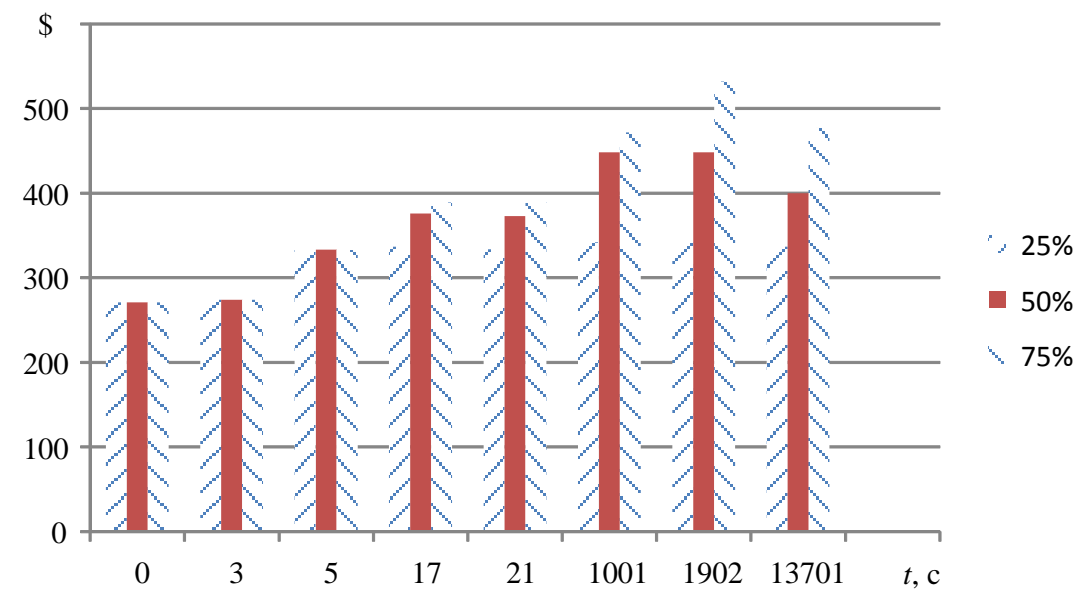

Рис. 1 - Динамика изменения текущей стоимости функционирования системы при различных сценариях

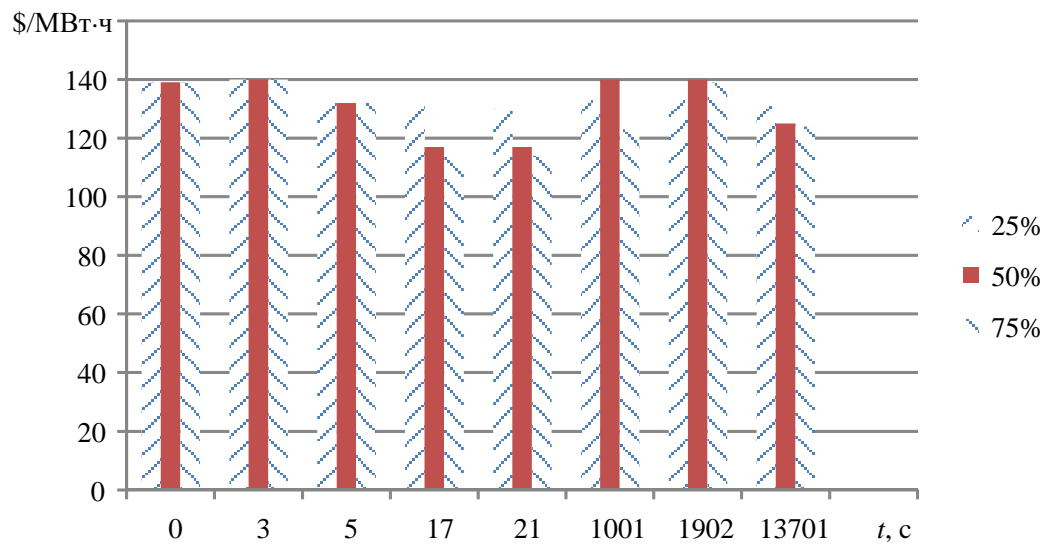

Рис. 2 - Динамика изменения удельной стоимости функционирования системы при различных сценариях

Выводы. Прежде всего следует отметить эффект от использования ДГУ. В сценарии с $25 \%$ дефицитом электроэнергии ДГУ позволили компенсировать почти $20 \%$ недостающей электроэнергии при увеличении стоимости всего на 0,0018\%. Время работы установок составило 100 с. В сценарии с 75\% дефицитом электроэнергии ДГУ позволили компенсировать 3\% недостающей электроэнергии при увеличении стоимости на 0,4\%. Время работы установок составило 1000 с. Также результаты свидетельствуют о том, что ДГУ не способны в значительной мере повлиять на изменение баланса всей энергосистемы. Мощность таких установок слишком мала, а их длительное использование делает их экономически нецелесообразными.

Следующий вывод - отсутствие в ЕЭС гидроэлектростанций существенно ухудшает возможности по балансировке системы. Если в сценарии с 25\% дефицитом энергии и наличием ГЭС суммарно мощностью 4 ГВт потребитель недополучил примерно 300 кВт॰ч, то в сценарии с отсутствующими ГЭС при тех же условиях потребитель недополучил уже 670000 кВт·ч. Это свидетельствует об очень важной роли мощных энергогенерирующих установок с малым временем пуска, таких как гидроагрегаты ГЭС и ГАЭС.

Вращающийся резерв также можно рассматривать как некую виртуальную энергоустановку с малым временем пуска. В рамках проведенного эксперимента вращающийся резерв позволил компенсировать от 50\% до $90 \%$ дефицита электроэнергии. В тоже время следует учитывать, что работа установок не на номинальной мощности связана с перерасходом топлива. На разных установках величина перерасхода различна, но может достигать $30 \%$ при текущей мощности установки составляющей $30 \div 40 \%$ от номинала. Поэтому организация вращающегося резерва всегда обходится дороже, чем работа установок на номинальной мощности.

Дублирование ВИЭ дизель-генераторными установками эквивалентной мощности позволяет полностью компенсировать возмущающие воздействия на ЕЭС со стороны ВИЭ. При этом стоимость эксплуатации не сильно отличается от варианта без дублирования. Так для сценария с 25\% дефицитом эта величина увеличилась на $8 \%$, а для сценария 75\% дефицита уменьшилась на 4\%. С другой стороны рыночная стоимость одной ДГУ мощностью 5 МВт сегодня составляет от 1,2 млн. евро. Для компенсации возмущения в сценарии 75\% дефицита потребовалось 1250 таких установок, что соответствует 1,5 млрд евро. Для сравнения ориентировочная стоимость 9 ГВт СЭС составить 7 млрд евро. 
Следующим важный результат показывает, что даже $25 \%$ дефицит электроэнергии который компенсируется

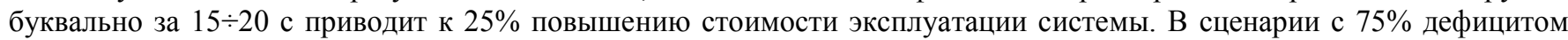
стоимость эксплуатации увеличилась в двое. При этом хотелось бы отметить, что легкая облачность снижает производство электроэнергии СЭС до 50\%. Рваная облачность может, как снижать в два раза производство энергии, так и повышать его в двое за счет отражения солнечных лучей от облаков. Густые облака, при которых отсутствует тень от объектов, снижают выработку энергии СЭС до минимума.

Также полученные результаты свидетельствуют о несостоятельности идей продавать «зеленую» энергию в страны ЕС. Из-за существенных колебаний частоты такая энергия просто не будет соответствовать требованиям к качеству.

Обоснование экономической эффективности ВИЭ по «зеленому» тарифу, не учитывает затраты на балансировку ЕЭС, а как показано в данной работе эти затраты могут составлять $100 \%$ от стоимости эксплуатации в нормальном режиме.

Единственным разумным подходом к использованию недиспетчеризуемых ВИЭ на данный момент видится жесткое ограничение на стабильность подключаемой к ЕЭС мощности. С каждой недеспечеризуемой установкой параллельно с ней должна вводиться диспечеризуемая установка такой же мощности. Организация либо частные предприниматели которые хотят продавать государству «зеленую» энергию должны предоставить годовой график производства электроэнергии. Любые отклонения от этого графика на величину более 5\% должны облагаться штрафами. Фактически это означает, что недиспетчеризуемый источник альтернативной энергии должен работать в комплексе с дизель-генераторными установками практически такой же мощности. Как показано в табл. 2 стоимость эксплуатации самой системы при этом не сильно изменится, но существенно возрастут капитальные затраты, что существенно снизит экономический эффект от использования ВИЭ. Адепты «зеленой» энергетики сегодня перекладывают роль этих дизель-генераторов на ЕЭС и тем самым убирают из уравнения экономической эффективности важную статью расходов.

\section{Список использованных источников}

[1] The International Energy Agency. Denmark. Balance 2017 [Электронный ресурс]. - Режим доступа: URL: https://www.iea.org/Sankey/\#?c=Denmark\&s=Balance

[2] Eurostat. [Электронный $\quad$ pecypc]. https://ec.europa.eu/eurostat/tgm/table.do?tab=table\&plugin=1\&language=en\&pcode=t2020_31.

[3] Energy numbers. Capacity factors at Danish offshore wind farms. [Электронный ресурс]. - Режим доступа: URL: http://energynumbers.info/capacity-factors-at-danish-offshore-wind-farms

[4] [Субботнее] Немецкое телевидение: Привыкайте к отключениям электричества [Электронный ресурс] / Тояма Токанава // AfterShock. 2018. - Режим доступа: URL: https://aftershock.news/?q=node/610440

[5] Павлов Г.М. Автоматическая частотная разгрузка энергосистем. Второе издание. - РАО «ЕЭС России» Центр подготовки кадров энергетики. СЗФ АО «ГВЦ Энергетики», 2002 г. 82 с.

[6] Добовий графік виробництва/споживання електроенергії // УКРЕНЕРГО. [Электронный ресурс]. - Режим доступа: URL: https://ua.energy/diyalnist/dyspetcherska-informatsiya/dobovyj-grafik-vyrobnytstva-spozhyvannya-e-e/

[7] Украина за год почти утроила мощности "зеленой" энергетики. [Электронный ресурс] // УНІАН. Інформаційне агентство. 2019 - Режим доступа: URL: https://www.unian.net/economics/energetics/10710915-ukraine-za-godpochti-utroila-moshchnosti-zelenoy-energetiki.html

\section{References}

[1]The International Energy Agency. Denmark. Balance 2017. - Available at: https://www.iea.org/Sankey/\#?c=Denmark\&s=Balance

[2] Eurostat - Available at: https://www.iea.org/Sankey/\#?c=Denmark\&s=Balance

[3] Energy numbers. Capacity factors at Danish offshore wind farms - Available at: http://energynumbers.info/capacityfactors-at-danish-offshore-wind-farms

[4] Tokanawa, T. (2018). [Subbotnee] Nemetskoe televidenie: Privykaite k otklucheniiam elektrichestva [[Saturday] German TV: Get Used To Power Outages]. AfterShock. - Available at: https://aftershock.news/?q=node/610440

[5] Pavlov, G. (2002). Avtomaticheskaia chastotnaia razgruzka [Automatic frequency unloading of power systems] [Text]. Second edition. - RAO "UES of Russia" Center for the training of energy personnel. NWF JSC "MCC Energy", 82 p.

[6] Dobovyi grafik vyrobnytsva/spojyvannia elektroenergii [UKRENERGO. Daily schedule of electricity production/consumption]. UKRENERGO. - Available at: https://ua.energy/diyalnist/dyspetcherskainformatsiya/dobovyj-grafik-vyrobnytstva-spozhyvannya-e-e/

[7] Ukraina za god pochti utroila moshchnosti «zelionoi» energetiki [Ukraine nearly tripled its green energy capacity over the year]. UNIAN. News Agency. 2019 - Available at: https://www.unian.net/economics/energetics/10710915-ukraineza-god-pochti-utroila-moshchnosti-zelenoy-energetiki.html 